\title{
111. Piecewise constant control of a precise measurement platform
}

\author{
R. Maskeliūnas', V. Maskeliūnas², A. Pauliukas³, L. Ragulskis ${ }^{4}$ \\ ${ }^{1,2}$ Vilnius Gediminas Technical University, Vilnius, Lithuania \\ ${ }^{3}$ Aleksandras Stulginskis University, Akademija, Kaunas District, Lithuania \\ ${ }^{4}$ Vytautas Magnus University, Kaunas, Lithuania \\ ${ }^{1}$ Corresponding author \\ E-mail: 1rimas.maskeliunas@vgtu.lt, ${ }^{2} v y t a u t a s . m a s k e l i u n a s @ s t u d . v g t u . l t,{ }^{3}$ arvydas.pauliukas@asu.lt, \\ 4.ragulskis@if.vdu.lt
}

Received 12 October 2016; received in revised form 15 December 2016; accepted 29 December 2016

DOI https://doi.org/10.21595/jme.2016.18145

\begin{abstract}
Control of a precise measurement platform is investigated in this paper. Piecewise constant control procedure on the basis of finite element in time and the method of least squares is proposed. It is demonstrated that the proposed technique can be used to follow not only a predetermined law of displacement variations, but also velocity variations. Computational experiments with a system comprising one degree of freedom are used to illustrate the efficiency of the proposed technique.
\end{abstract}

Keywords: measurement platform, finite element, piecewise constant control.

\section{Introduction}

Control of a precise measurement platform is investigated. Model having one degree of freedom is used. Control procedure on the basis of finite element in time and the method of least squares is proposed. Piecewise constant control force is obtained. The proposed method of control is based on the material presented in [1-5]. Related problems are investigated in [6-11]. On the basis of performed investigations for various values of parameters the necessity to follow not only the desirable displacement, but also the desirable velocity is shown.

\section{Control of a precise measurement platform}

Motion of a precise measurement platform is approximated by the standard one-dimensional differential equation:

$m \ddot{u}+c \dot{u}+k u=f$,

where $m$ is the mass of the platform, $u$ is the displacement, $c$ is the coefficient of viscous damping, $k$ is the coefficient of stiffness, $f$ is the external force, upper dot denotes differentiation with respect to time.

Linear interpolation of displacements on the basis of the method of finite elements yields:

$\left[\begin{array}{cc}-\frac{m}{T}-\frac{c}{2}+k \frac{T}{3} & \frac{m}{T}+\frac{c}{2}+k \frac{T}{6} \\ \frac{m}{T}-\frac{c}{2}+k \frac{T}{6} & -\frac{m}{T}+\frac{c}{2}+k \frac{T}{3}\end{array}\right]\left\{\begin{array}{l}u_{0} \\ u_{T}\end{array}\right\}=\left\{\begin{array}{l}\frac{T}{2} f+m \dot{u}_{0} \\ \frac{T}{2} f-m \dot{u}_{T}\end{array}\right\}$,

where $T$ is the time step, the subscript 0 corresponds to the value at the beginning of the time step, the subscript $T$ corresponds to the value at the end of the time step. Calculations of displacements by using this finite element are based on the following equation:

$u_{T}=\frac{B}{A}$ 
where:

$B=f \frac{T}{2}-\left(-\frac{m}{T}-\frac{c}{2}+k \frac{T}{3}\right) u_{0}+m \dot{u}_{0}$

$A=\frac{m}{T}+\frac{c}{2}+k \frac{T}{6}$.

Calculations of velocity by using this finite element are based on the following equation:

$\dot{u}_{T}=\frac{1}{m}\left(f \frac{T}{2}-\left(\frac{m}{T}-\frac{c}{2}+k \frac{T}{6}\right) u_{0}-\left(-\frac{m}{T}+\frac{c}{2}+k \frac{T}{3}\right) u_{T}\right)$.

The following equation for displacement is obtained:

$u_{T}=G f+H$,

where:

$G=\frac{\frac{T}{2}}{A}$,

$H=\frac{1}{A}\left(-\left(-\frac{m}{T}-\frac{c}{2}+k \frac{T}{3}\right) u_{0}+m \dot{u}_{0}\right)$.

The following equation for velocity is obtained:

$\dot{u}_{T}=\bar{G} f+\bar{H}$,

where:

$\bar{G}=\frac{1}{m}\left(\frac{T}{2}-\left(-\frac{m}{T}+\frac{c}{2}+k \frac{T}{3}\right) G\right)$,

$\bar{H}=\frac{1}{m}\left(-\left(\frac{m}{T}-\frac{c}{2}+k \frac{T}{6}\right) u_{0}-\left(-\frac{m}{T}+\frac{c}{2}+k \frac{T}{3}\right) H\right)$.

The following error function is introduced:

$\varepsilon=\frac{\lambda_{1}}{2}\left(u_{T}-\bar{u}\right)^{2}+\frac{\lambda_{2}}{2}\left(\dot{u}_{T}-\overline{\dot{u}}\right)^{2}$,

where $\lambda_{1}$ and $\lambda_{2}$ are the weighing parameters, $\bar{u}$ is the desirable displacement, $\overline{\dot{u}}$ is the desirable velocity.

This function can be rewritten as:

$\varepsilon=\frac{\lambda_{1}}{2}(G f+H-\bar{u})^{2}+\frac{\lambda_{2}}{2}(\bar{G} f+\bar{H}-\overline{\dot{u}})^{2}$.

By minimizing the error, it is obtained:

$\lambda_{1} G(G f+H-\bar{u})+\lambda_{2} \bar{G}(\bar{G} f+\bar{H}-\overline{\dot{u}})=0$.

Thus, the excitation is calculated as: 
$f=-\frac{\lambda_{1} G(H-\bar{u})+\lambda_{2} \bar{G}(\bar{H}-\overline{\dot{u}})}{\lambda_{1} G^{2}+\lambda_{2} \bar{G}^{2}}$.

\section{Computational experiments for the control of a precise measurement platform}

The following parameters of the investigated precise measurement platform are assumed: $m=1, c=0.1, k=1$.

Desirable motion is assumed as:

$\bar{u}=4 \sin \omega t$,

$\overline{\dot{u}}=4 \omega \cos \omega t$,

where $\omega$ is the angular frequency of harmonic motion, $t$ is the time variable.

Initial conditions are assumed as:

$u(0)=0$,

$\dot{u}(0)=4 \omega$.

Time step is assumed as:

$T=\frac{\frac{2 \pi}{\omega}}{40}$.

Displacement control is performed by assuming $\lambda_{1}=1$ and $\lambda_{2}=0$. Displacement and velocity control is performed by assuming $\lambda_{1}=1$ and $\lambda_{2}=1$.

Control of the precise measurement platform is investigated for various values of frequency of desirable motion. Typical results of control of the precise measurement platform when $\omega=0.5$ are presented in detail further.

\subsection{Displacement control}

Displacement control is performed by assuming $\lambda_{1}=1$ and $\lambda_{2}=0$.

Variation of the control force is presented in Fig. 1.

Variations of displacement and velocity are presented in Fig. 2.

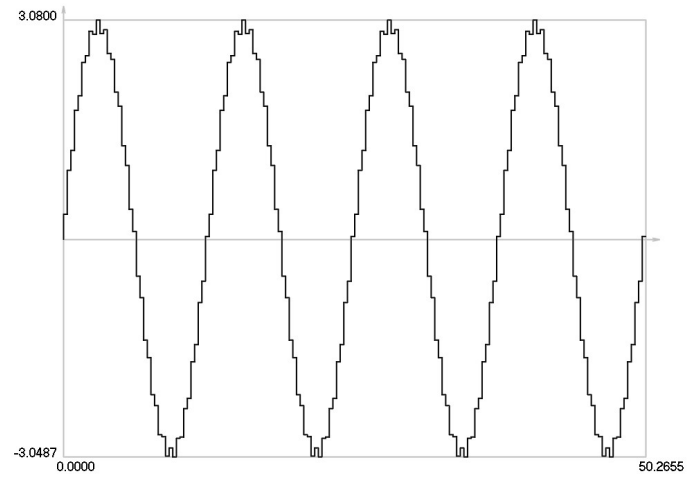

Fig. 1. Control force as function of time

Phase trajectory of velocity as a function of displacement is presented in Fig. 3.

From the presented graphical representation, it is seen that the control force has undesirable high frequency oscillations. Thus, their investigation for one period of steady state motion is 
performed in more detail. Variation of the control force over one period of steady state motion is presented in Fig. 4.

By using the method of collocation - least squares the first harmonic of the control force over one period of steady state motion is determined. Midpoints of time steps are used as collocation points. The first harmonic of the control force over one period of steady state motion is presented in Fig. 5.

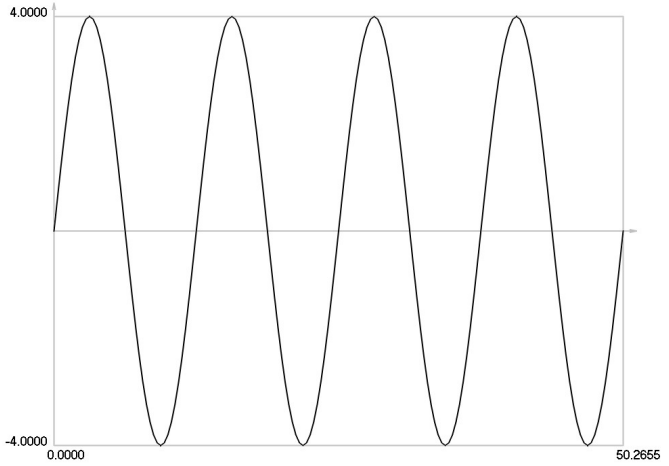

a) Displacement as function of time

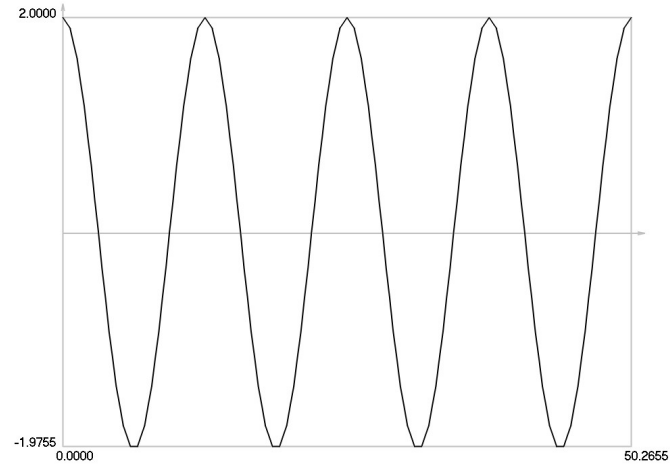

b) Velocity as function of time

Fig. 2. Displacement and velocity as functions of time

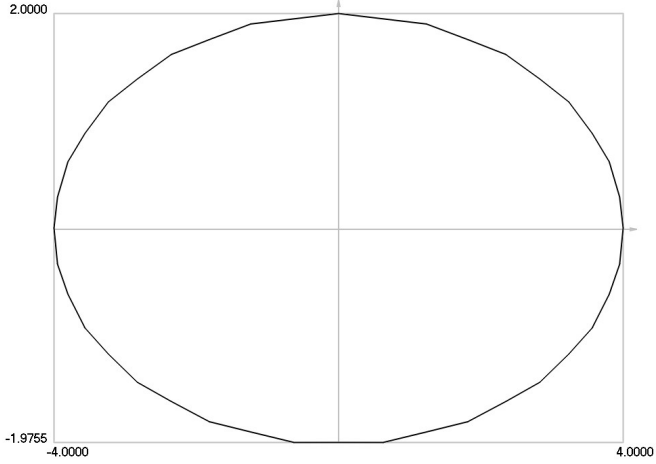

Fig. 3. Phase trajectory of velocity as a function of displacement

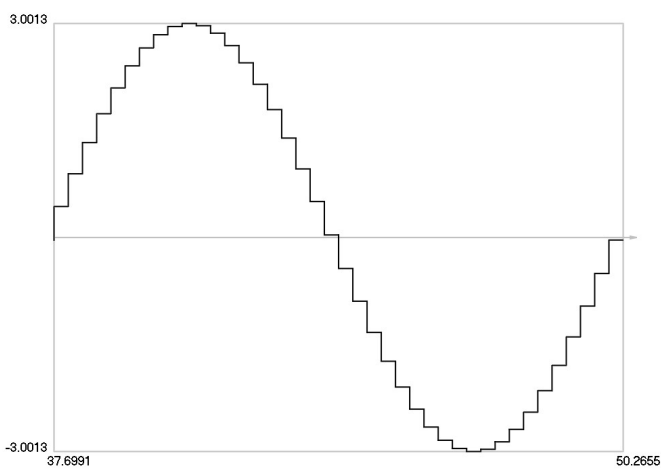

Fig. 5. The first harmonic of the control force as function of time over one period of steady state motion

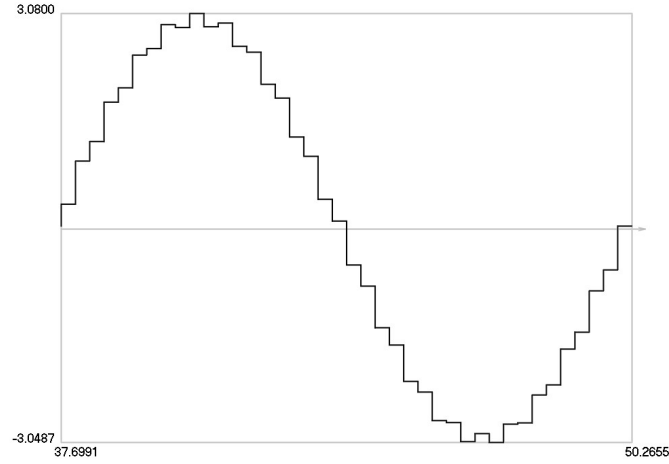

Fig. 4. Control force as function of time over one period of steady state motion

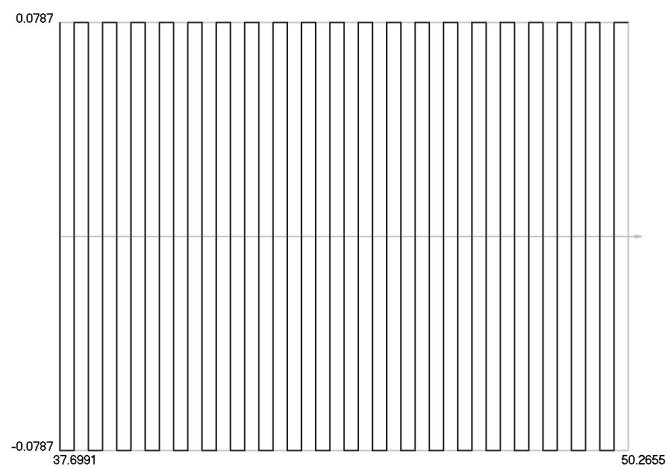

Fig. 6. The error of the control force as function of time over one period of steady state motion 
The error (that is deviation from the first harmonic) of the control force over one period of steady state motion is presented in Fig. 6.

From the presented results, it is seen that the variation of the control force is not acceptable and thus it cannot be recommended to perform only displacement control.

\subsection{Displacement and velocity control}

Computational experiments for the displacement and velocity control are performed by assuming $\lambda_{1}=1$ and $\lambda_{2}=1$. Variation of the control force is presented in Fig. 7 .

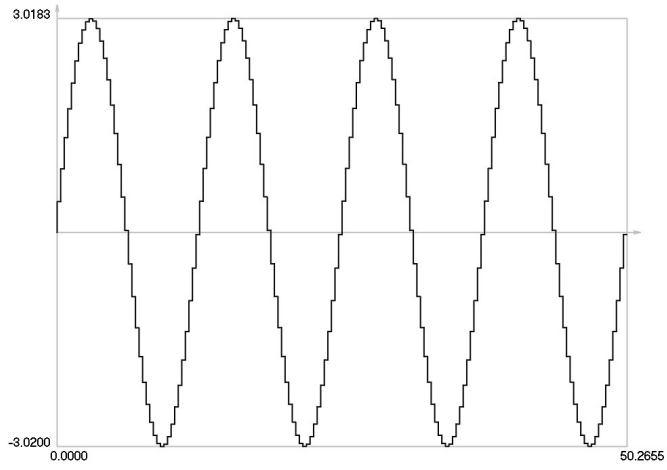

Fig. 7. Control force as function of time

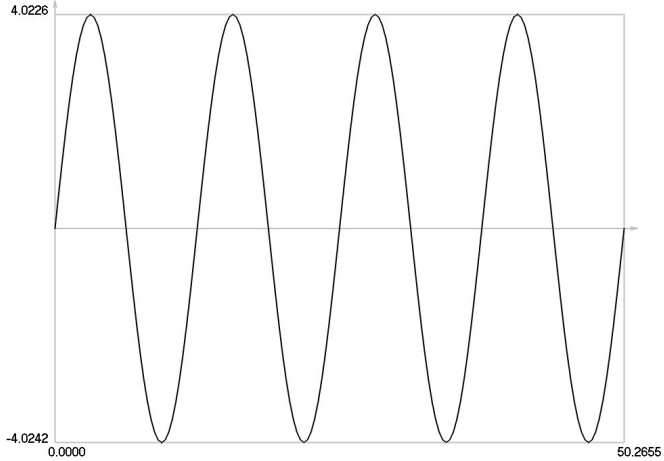

a) Displacement as function of time

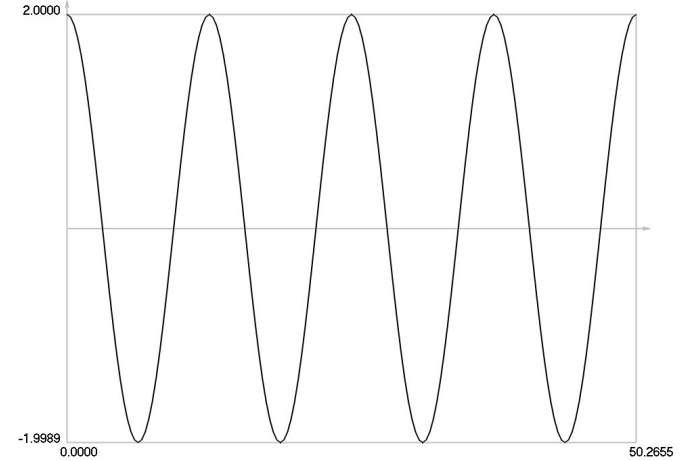

b) Velocity as function of time

Fig. 8. Displacement and velocity as functions of time

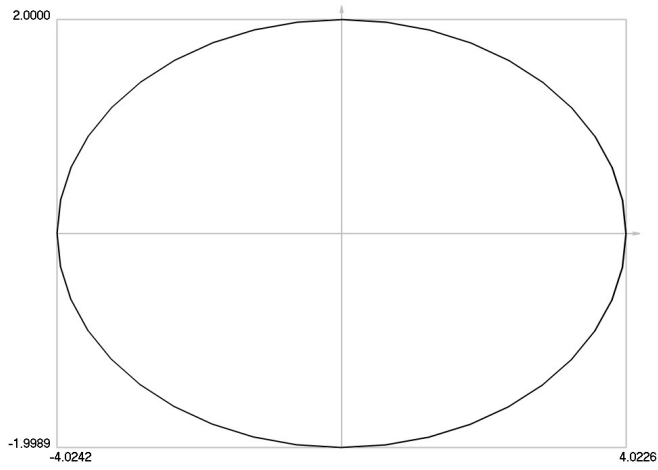

Fig. 9. Phase trajectory of velocity as a function of displacement 
Variations of displacement and velocity are presented in Fig. 8.

Phase trajectory of velocity as a function of displacement is presented in Fig. 9.

From the presented results, it is seen that the variation of the control force is acceptable (high frequency oscillations are not observed in the obtained results) and thus displacement and velocity control is recommended for engineering applications where the stabilization of harmonic oscillations is required.

\section{Conclusions}

Control of a precise measurement platform is investigated. Model having one degree of freedom is used. Control procedure on the basis of finite element in time and the method of least squares is proposed. Piecewise constant control is investigated.

From the presented results, it is seen that the resulting variations of displacement and velocity as functions of time are acceptable for both problems: problem of displacement control and problem of control of both displacement and velocity. But piecewise constant variation of the control force has high frequency vibrations when only displacement control is performed. Computational simulations demonstrate the necessity to follow not only the desirable displacement, but also the desirable velocity of the controlled system. Experimental implementation of the proposed technique is a definite objective of future research.

\section{References}

[1] Johnson Claes Numerical Solution of Partial Differential Equations by the Finite Element Method. Cambridge University Press, New York, 1987.

[2] Larson Mats G., Bengzon Fredrik The Finite Element Method: Theory, Implementation, and Applications. Texts in Computational Science and Engineering, Vol. 10, 2013.

[3] Rao S. The Finite Element Method in Engineering, 5th Edition. Elsevier, Butterworth-Heinemann, 2010, p. 728.

[4] Inman D. J. Vibration with Control, Measurement, and Stability. Prentice-Hall, New Jersey, 1989.

[5] Zimmerman William B. J. Series on Stability, Vibration and Control of Systems, Series A: Volume 18. Multiphysics Modeling with Finite Element Methods. World Scientific, 2006, p. 432.

[6] Levy S., Wilkinson J. P. D. The Component Element Method in Dynamics with Application to Earthquake and Vehicle Engineering. McGraw-Hill, New York, 1976.

[7] Bathe K. J. Finite Element Procedures in Engineering Analysis. Prentice-Hall, New Jersey, 1982.

[8] Bathe K. J., Wilson E. L. Numerical Methods in Finite Element Analysis. Stroiizdat, Moscow, 1982, (in Russian).

[9] Bolotin V. V. Vibrations in Engineering. Handbook, Vol. 1, Mashinostroienie, Moscow, 1978, (in Russian).

[10] Lalanne M., Berthier P., Der Hagopian J. Mechanical Vibrations for Engineers. John Wiley and Sons, New York, 1984.

[11] Thomson W. T. Theory of Vibration with Applications. Prentice-Hall, New Jersey, 1981. 